\title{
Identifying and Correcting Biases in Localized Downscaling Estimates of Daily Precipitation Return Values
}

\section{Mark Risser ( $\square$ mdrisser@lbl.gov )}

Lawrence Berkeley National Laboratory: E O Lawrence Berkeley National Laboratory https://orcid.org/0000-0003-1956-1783

\section{Daniel Feldman}

Lawrence Berkeley Laboratory: E O Lawrence Berkeley National Laboratory

\section{Michael Wehner}

Lawrence Berkeley National Laboratory: E O Lawrence Berkeley National Laboratory

\section{David Pierce}

University of California San Diego Scripps Institution of Oceanography

Jeff Arnold

Corps: US Army Corps of Engineers

\section{Research Article}

Keywords: Extreme precipitation, Local impacts, Localized Constructed Analogs, Scaling factor

Posted Date: August 9th, 2021

DOl: https://doi.org/10.21203/rs.3.rs-610939/v1

License: (c) (1) This work is licensed under a Creative Commons Attribution 4.0 International License. Read Full License

Version of Record: A version of this preprint was published at Climatic Change on December 1st, 2021. See the published version at https://doi.org/10.1007/s10584-021-03265-z. 


\title{
Identifying and correcting biases in localized downscaling estimates of daily precipitation return values
}

\author{
3 Authors blinded
}

5 Received: date / Accepted: date

6 Abstract Extreme precipitation events are a major cause of economic damage and disrup7 tion, and need to be addressed for increasing resilience to a changing climate, particularly 8 at the local scale. Practitioners typically want to understand local changes at spatial scales much smaller than the native resolution of most Global Climate Models, for which downscaling techniques are used to translate planetary-to-regional scale change information to local scales. However, users of statistically downscaled outputs should be aware that how the observational data used to train the statistical models is constructed determines key properties of the downscaled solutions. Specifically for one such downscaling approach, when considering seasonal return values of extreme daily precipitation, we find that the Localized Constructed Analogs (LOCA) method produces a significant low bias in return values due to choices made in building the observational data set used to train LOCA. The LOCA low biases in daily extremes are consistent across event extremity, but do not degrade the overall performance of LOCA-derived changes in extreme daily precipitation. We show that the low bias in daily extremes is a function of a time-of-day adjustment applied to the training data and the manner of gridding daily precipitation data. The effects of these choices are likely to affect other downscaling methods trained with observations made in the same way. The results developed here show that efforts to improve resilience at the local level using extreme precipitation projections can benefit from using products specifically created to properly capture the statistics of extreme daily precipitation events.

25 Keywords Extreme precipitation - Local impacts - Localized Constructed Analogs ·

26 Scaling factor

\section{$27 \quad 1$ Introduction}

Address(es) of author(s) should be given 
nonstationarity of those distributions, which are among the most important state variables for understanding climate change threats and impacts (Milly et al. 2008, Hallegatte, 2009, Engle 2011). The climate science community has engaged in major international efforts through the Coupled Model Intercomparison Project (CMIP) exercises (e.g., Taylor et al., 2012, Eyring et al. 2016) to assess and understand changes in the planetary-to-regionalscale distributions of temperature and precipitation, and the associated analyses from that work are a key part of most applications of global-scale climate information for understanding and preparing for local impacts. These Global Climate and Earth System Models (GCMs and ESMs) use numerical representations of physical processes in Earth's climate system to project, among other effects, a range of responses of Earth's hydrological cycle as it adjusts to the warming resulting from specified concentration or emissions pathways.

However, GCMs and ESMs and the experiments they run for CMIP are not designed to depict local change. Computational limitations still require that most of the productionclass GCMs and ESMs used in the CMIP experiments use $100 \mathrm{~km}$ (or larger) geospatial grids, which can be much larger than the scale of the decisions that practitioners must make concerning local impacts. One widely-used approach to make native-scale GCM output more useful at finer spatial scales is to downscale the GCM outputs. Downscaling methods range across a continuum from empirical-statistical (e.g., Wood et al., 2004; Abatzoglou and Brown, 2012, Stoner et al. 2013, Pierce et al., 2014), to fully dynamical depending on the extent to which physical processes are represented in the downscaling approach (e.g., Giorgi and Gutowski Jr. 2015), with hybrid methods between those two (e.g., Gutmann et al., 2016). However, the wide range of downscaling methods often produces a range of model results for historical and projected future conditions, which can complicate long-term planning efforts (Martinich and Crimmins, 2019, Lopez-Cantu et al. 2020). Therefore, a better understanding of why downscaled solutions diverge is important to practitioners as well as climate scientists.

Practitioners using downscaled projections to describe and understand atmospheric and land-surface impacts at individual fine-scale locations should consider multiple questions (e.g., Barsugli et al. 2013, Vano et al., 2018), including the tolerance for model spread across key variables and the level of confidence and fidelity to known processes required for their fine-scale studies. These questions arise irrespective of whether practitioners directly apply downscaled fields to specific locations or indirectly apply downscaled outputs through approaches such as decision-scaling, in which a stochastic assessment of risk is combined with insights derived from climate projections at some scale to determine the likelihood of changes in events of particular interest at a given location (e.g., Brown et al. 2012). When the environmental variable involves daily precipitation extremes, directly using native GCM or ESM outputs with $\geq 100 \mathrm{~km}$ spatial resolution can often be excluded at the start of many practitioners' studies since the scales of their decisions are so much finer. However, downscaling extremes in daily precipitation is one of the key challenges for statistical downscaling methods (Chen and Zhang, 2021), particularly since the gridded daily precipitation data products used for training are well-known to diminish variability and mute extremes (King et al. 2013, Risser et al., 2019c). The important point is that any data set or technique used should be able to capture extremes, which in principle can be accomplished by a variety of methods. One such example that can be tailored to reproduce and analyze extremal behaviour is stochastic weather generators (SWGs; Vrac et al. 2007; Ayar et al. 2020), although coherency across space and variables is still a challenge for SWGs. Unfortunately, many of these approaches (including SWGs) require domain-specific scientific and statistical expertise that can be infeasible for practitioners to implement. It is far more practical for practitioners to analyze existing downscaled products than to, e.g., implement and 
run stochastic weather generators, and they can benefit from understanding when and where such products are appropriate for a particular problem of interest. To that end, we explore here how known biases in gridded daily precipitation products translate into corresponding biases in downscaling solutions.

In this paper, we demonstrate how the process of constructing observational data sets for training the empirical-statistical downscaling methods has a direct influence on the downscaling solutions, particularly for the problem of long-period return values of daily precipitation extremes. Specifically, we explore in detail the influence of training data on the Localized Constructed Analogs (LOCA) statistical downscaling method (Pierce et al. 2014), which used the daily gridded precipitation data set Livneh et al. (2015a b) (henceforth L15) as training data, and show how using a product specifically constructed to accurately capture pointwise extreme precipitation statistics can give a more useful depiction of such extremes than the standard gridded daily precipitation product used to train the statistical models.

The paper proceeds as follows: in Section 2, we describe the data sets (observational and modeled) and methods for comparing estimates of seasonal return values for daily precipitation. In Section 3, we present our results for an illustrative set of specific locations, investigate two primary reasons for biases in the gridded daily product used for training the statistical downscaling solutions, derive a new data product of scaling factors that allows users of LOCA products derived from CMIP5 model outputs to adjust return value estimates, and apply the scaling factor to our illustrative locations. Finally, in Section 4 we conclude with the implications of these findings and suggestions for practitioners focused on location-specific analyses.

\section{Data and methods}

\subsection{Observational data products}

Livneh daily gridded product The Livneh et al.(2015a b) daily gridded product provides estimates of daily precipitation at 16th degree spatial resolution over much of North America for 1950-2013. L15 is a widely used data product, and is relevant here because it is used as training data for the LOCA downscaling method. The L15 data product uses in situ measurements of daily total precipitation (over CONUS, the input data are from the Global Historical Climatology Network; Menne et al. 2012) to create a daily gridded product in two steps. First, for each day, the station measurements are interpolated to a $1 / 16^{\circ}$ or $\sim 6 \mathrm{~km}$ highresolution grid using the SYMAP algorithm (Shepard, 1968, 1984), which is an inversedistance weighting approach. Second, the interpolated data are multiplied by a monthly scaling factor determined by the ratio of its mean monthly baseline climatology (1981-2010) and the mean monthly climatology from the same period of the topographically-aware PRISM data product (Daly et al. 1994, 2008). The L15 data product is largely an extension of an earlier version of the data set (Livneh et al. 2013, henceforth L13); all time-of-day splitting issues (see Section 3.2 were first raised in L13 and then inherited by L15.

Risser climatological gridded product Gridded daily products like L15 have been identified recently (King et al., 2013, Gervais et al., 2014, Timmermans et al., 2019) as inappropriate for characterizing extreme local precipitation since daily precipitation is known to exhibit fractal scaling (see, e.g., Lovejoy et al. 2008, Maskey et al., 2016, and numerous references therein). For this reason, any spatial averaging during the gridding process will diminish variability and extreme values in the original observations. As an alternative, Risser et al. 
(2019c) develop a "probabilistic" gridded data product that is appropriate for climatological analyses and is specifically designed to characterize local measurements of extreme precipitation. The Risser et al. (2019a) data product (henceforth R19) preserves the extreme statistics of weather station measurements by first calculating extreme statistics at each station and then interpolating these statistics using a data-driven technique to a $1 / 4^{\circ}$ or $\sim 25 \mathrm{~km}$ grid. Furthermore, R19 contains uncertainty quantification in the form of a bootstrap or resampling-based ensemble of estimates. The benefits of the R19 data set (as documented in Risser et al. 2019c) include (1) the spatial aspect of the statistical analysis borrows strength from neighboring stations when estimating the extreme statistics at each station and (2) this borrowing of strength reduces the uncertainty such that the signal-to-noise ratio of the underlying spatial signal is increased. Compared to a single-station analysis that estimates extreme statistics of daily precipitation at each station independently of all others, the R19 product has small root mean squared error in 20-year return values (ranging from $5.5 \mathrm{~mm}$ in DJF to $11.2 \mathrm{~mm}$ in JJA as a CONUS average; much of this "error" is smoothing over observational uncertainty) and significantly smaller bootstrap standard errors (ranging from a reduction of $40 \%$ in DJF to more than $50 \%$ in JJA).

It is important to emphasize that R19 does not attempt to grid daily precipitation, and hence only provides climatological summaries (i.e., return values) with measures of uncertainty and so cannot be used to train traditional statistical downscaling methods in place of gridded products. The implication, however, is that daily extremes in precipitation as represented in R19 are not "smeared" over space, which is a fundamental property of any data product that attempts to interpolate daily precipitation without explicitly accounting for fractal scaling in precipitation (including L15). This includes other ensemble data products (e.g. Newman et al. 2015), which provide measures for estimating uncertainty but still diminish extreme values and variability by gridding daily precipitation data.

Statistically downscaled data products As previously mentioned, the L13 and L15 datasets have many applications, including as a training dataset for the Localized Constructed Analogs (LOCA) statistical downscaling method (Pierce et al. 2014). LOCA has been used to downscale climate model outputs including those from the CMIP5 archive, and LOCA-downscaled CMIP5 model products for historical and future emissions scenarios have been widely used, including as the basis for projections of change in temperature and precipitation across the Conterminous United States in the 4th National Climate Assessment (Wuebbles et al. 2017). Those LOCA outputs were downscaled from 32 GCMs and have a daily timestep for the period 1950-2100. Here we use GCM outputs downscaled from the CMIP5 historical period (1950-2005), for the 31 CMIP5 models shown in Supplemental Table 1.

LOCA is a constructed analog method for downscaling. In the constructed analog approach, fine-scale observations are first coarsened to the GCM grid, then the 30 coarsened observed days that best match the specific GCM day being downscaled are identified. These are termed the analog days. Traditional constructed analog downscaling then calculates the weighted average of the 30 analog days that best reproduces the specific GCM day being downscaled. The 30 weights are then applied to the 30 original high-resolution observational days, and the resulting weighted average is the high-resolution downscaled solution. However this averaging has the drawback that extremes are muted and (for precipitation) drizzle is generated in the downscaled field. LOCA avoids this by starting with the 30 analog days, but then using only the single best-matching analog day of the 30 in a $1 \times 1$ degree latitudelongitude box around the point being downscaled to. With less averaging, extreme values as represented in the training data are better preserved in the downscaled field and extraneous drizzle is not generated. Details are given in Pierce et al. (2014). 


\subsection{Return value assessment}

Our key objective with this paper is to compare estimates of daily precipitation return values at specific locations from LOCA and R19 in order to demonstrate how confidence in estimates of extreme daily precipitation return values can be enhanced by consulting a product that is specifically constructed on the basis of extreme value analysis. Return values (sometimes referred to as a return level) for daily precipitation define a threshold for what is considered an extreme or severe daily precipitation total at a given location. As such, return values are an important quantity for assessing local risk and designing infrastructure through quantifying the probability of rare events, including estimates for events that have not occurred in a particular observational period. Return values are defined for a particular "return period" $r$, where often $r=10,20,50$, or 100 years, which specifies the event rarity: for example, in any given year, the probability of exceeding the threshold defined by the 20 -year return value is $1 / 20=0.05$. In order to compare return value estimates between R19 and LOCA, we select the nearest R19 grid cell (25 km resolution) and LOCA grid cell (6 km resolution) for each of a set of locations across CONUS, focusing on US Department of Defense facilities where practitioners are planning for hydroclimatological extremes by considering downscaling solutions (Moss, 2017). We evaluate the influence of the different spatial scales of these data products in the Supplemental Materials.

The R19 data product directly provides return value estimates for daily precipitation at each grid cell in each of the four three-month seasons (DJF, MAM, JJA, and SON), and hence contains the extreme statistics of interest. To calculate the corresponding seasonal quantities for the LOCA solutions, we apply an extreme value analysis to the time series of daily precipitation at each matched grid cell. First, for each grid cell in each season/year we calculate the maximum daily precipitation total $(\mathrm{mm})$, commonly referred to as Rx1Day. Next, we characterize the extreme climatology for each grid cell using the Generalized Extreme Value (GEV) family of distributions, which is a statistical modeling framework for the maxima of a process over a specified time interval or "block"; here, the three-month seasons. Coles (2001) (Theorem 3.1.1, page 48) shows that when the number of measurements per block is large enough, the cumulative distribution function (CDF) of the seasonal Rx1Day in year $t$ and grid cell $\mathbf{g}$, denoted $Y_{t}(\mathbf{g})$, can be approximated by a member of the GEV family

$$
G_{\mathbf{g}, t}(y) \equiv \mathbb{P}\left(Y_{t}(\mathbf{g}) \leq y\right)=\exp \left\{-\left[1+\xi_{t}(\mathbf{g})\left(\frac{y-\mu_{t}(\mathbf{g})}{\sigma_{t}(\mathbf{g})}\right)\right]^{-1 / \xi_{t}(\mathbf{g})}\right\},
$$

defined for $\left\{y: 1+\xi_{t}(\mathbf{g})\left(y-\mu_{t}(\mathbf{g})\right) / \sigma_{t}(\mathbf{g})>0\right\}$. The GEV family of distributions (1) is characterized by three statistical parameters: the location parameter $\mu_{t}(\mathbf{g}) \in \mathscr{R}$, which describes the center of the distribution; the scale parameter $\sigma_{t}(\mathbf{g})>0$, which describes the spread of the distribution; and the shape parameter $\xi_{t}(\mathbf{g}) \in \mathscr{R}$. The shape parameter $\xi_{t}(\mathbf{g})$ is the most important for determining the qualitative behavior of the distribution of daily rainfall at a given location. If $\xi_{t}(\mathbf{g})<0$, the distribution has a finite upper bound; if $\xi_{t}(\mathbf{g})>0$, the distribution has no upper limit; and if $\xi_{t}(\mathbf{g})=0$, the distribution is again unbounded and the CDF (1) is interpreted as the limit as the shape parameter $\xi_{t}(\mathbf{g})$ approaches zero (Coles, 2001 .

To account for changes over time in the distribution of extreme precipitation and temperature we use a simple trend model wherein the location parameter varies linearly in time, i.e., $\mu_{t}(\mathbf{g})=\mu_{0}(\mathbf{g})+\mu_{1}(\mathbf{g}) t$, while the shape and scale parameters are constant over time, i.e., $\sigma_{t}(\mathbf{g}) \equiv \sigma(\mathbf{g})$ and $\xi_{t}(\mathbf{g}) \equiv \xi(\mathbf{g})$. Using just four parameters to model changes over time 
in Eq. 1 at each grid cell is a simplistic representation of the temporal evolution of extreme precipitation, since in reality the distribution may be shifting nonlinearly (e.g., due to nonlinear changes in external forcing) with possibly nonconstant variability. However, Risser et al. (2019b) explored both (a) including nonlinear trends in the location parameter and (b) allowing the shape and scale parameters to change over time, and found that the simpler model described by a linear trend in location with constant scale and shape performed as well (in a statistical sense) as either of these alternative characterizations. Hence, we argue that this statistical model is an appropriate way to characterize changes in extreme precipitation over CONUS. We use the climextRemes package for $\mathrm{R}$ (Paciorek. 2016) to obtain maximum likelihood estimates (MLEs) of the GEV parameters $\left\{\widehat{\mu}_{0}(\mathbf{g}), \widehat{\mu}_{1}(\mathbf{g}), \widehat{\sigma}(\mathbf{g}), \widehat{\xi}(\mathbf{g})\right\}$, independently for each grid cell of interest and quantify uncertainty via the block bootstrap (see, e.g., Risser et al., 2019c). The MLEs and the bootstrap estimates can be used to derive estimates of the seasonal climatological $r$-year return value in year $t$, denoted $\widehat{\phi}_{t, r}(\mathbf{g})$, which can be calculated as:

$$
\widehat{\phi}_{t, r}(\mathbf{g})= \begin{cases}\widehat{\mu}_{0}(\mathbf{g})+\widehat{\mu}_{1}(\mathbf{g}) t-\frac{\widehat{\sigma}(\mathbf{g})}{\widehat{\xi}(\mathbf{g})}\left[1-\{-\log (1-1 / r)\}^{-\widehat{\xi}(\mathbf{g})}\right], & \widehat{\xi}(\mathbf{g}) \neq 0 \\ \widehat{\mu}_{0}(\mathbf{g})+\widehat{\mu}_{1}(\mathbf{g}) t-\widehat{\sigma}(\mathbf{g}) \log \{-\log (1-1 / r)\}, & \widehat{\xi}(\mathbf{g})=0\end{cases}
$$

(Coles, 2001). In summary, after conducting the above analysis, we can calculate MLEs of the climatological Rx1Day $r$-year return value in year $t \widehat{\phi}_{t, r}(\mathbf{g})$ and block bootstrap estimates $\left\{\widehat{\phi}_{t, r, b}(\mathbf{g}): b=1, \ldots, 250\right\}$ in each season, and for each of the LOCA-downscaled models. Again, the R19 data product yields these return value MLEs and bootstrap estimates directly. The bootstrap estimates are used to calculate a 95\% confidence interval using the "basic bootstrap" method (see, e.g., Paciorek et al. 2018).

In order to compare the LOCA solutions versus R19, in addition to the return values we consider three quantities for each geospatial location: (1) the relative difference in return value (LOCA minus R19, divided by R19); (2) the 65-year trend (1951-2005) in return values, as determined by $\widehat{\mu}_{1}(\mathbf{g})$; and (3) the linear trend in the relative difference between LOCA and R19 as a function of the return period $r$. For each of these quantities, at a specific location we first tally the number of LOCA solutions that differ from R19 (as determined by whether the $95 \%$ confidence intervals overlap) and, if there appear to be meaningful differences between the LOCA solutions and R19, we state differences between the LOCA average and R19.

\section{Results}

\subsection{Return value comparison}

To assess the performance of statistical downscaling solutions for location-specific analysis of extreme precipitation, we use, as a case study, the subset of United States Department of Defense (DoD) installations within the CONUS that serve as foci for environmental research supported by the Strategic Environmental Research and Development Program (SERDP) and Environmental Security Technology Certification Program (ESTCP). We specifically focus on a total of 163 installations that are affiliated with the Air Force, Army, Coast Guard, Marine Corps, National Guard, or the Navy, and have a unique ZIP code; the geographic distribution of these sites is shown in Figure 1 These specific locations are chosen for our analysis because practitioners who are assessing the risks at each site from climate-changed 
temperature and precipitation patterns would benefit from high-confidence, localized projections of statistical distributions of these quantities (Moss 2017).

As an illustration of our methodology, we first choose seven facilities that represent a variety of climatological regions in the CONUS; again see Figure 1 For each facility, we compare estimates of wintertime (DJF) return values for daily maximum precipitation (Rx1Day). Recall that the $r$-year return value summarizes the event (precipitation threshold) that is expected to be exceeded once every $r$ years (on average); see Figure 2(a), which shows the return values and 95\% confidence band for R19 and the average of the 31 LOCAdownscaled CMIP5 models. Across the board, the LOCA average is significantly lower than R19. To specifically quantify these differences, Figure 2 b) shows the relative difference between the LOCA average and R19: these differences are all negative (again highlighting the low bias) and range between $-30 \%$ and $-50 \%$, with the $95 \%$ confidence band showing that the differences are significant. This remains the case when considering the LOCA outputs derived from individual models: Figure 2 (c) shows the number of individual LOCA solutions that are significantly different from R19. For less rare events (up to the 20-year event), every LOCA solution differs from R19 except at Fort Hood. For more extreme events, the uncertainty is large enough that fewer of the LOCA solutions differ from R19, but a majority still show significant differences. In spite of the large differences between R19 and the LOCA average, the percent differences are relatively consistent across return period $r$. In other words, the relative errors (percent) in semi-rare events, e.g., the 1 -in-5 year event, are approximately the same (after accounting for uncertainty) as much rarer events, e.g., the 1-in-50 year event. The strip text in Figure 2 (b) shows the trend in the percent difference as a function of return period $r$ (percent per 10-year increase in return period); only Tyndall Air Force Base has a statistically significant trend in the difference, although the trend is very small (less than $1 \%$ per 10-year increase in return period).

Having explored how the various data products compare in terms of the climatology of extreme precipitation (i.e., return values), we next move on to assess how the products compare in terms of how they quantify changes in extremes over time, specifically with respect to linear trends over 1951-2005. Recall that our GEV analysis quantifies changes over time in terms of a linear trend in the location parameter. Focusing on 20-year return values, we convert this trend into $\mathrm{mm} / \mathrm{century}$ and again compute a $95 \%$ basic bootstrap confidence interval. Figure 2]d) compares the trend estimates at each facility for the LOCA average and R19: the uncertainty in the trends themselves are generally quite large, such that in most cases the trends are non-significant across data source; this agrees with significance statements regarding observed trends in GHCN return values from Risser et al. (2019b). Consequently, the differences in 1951-2005 trends across the LOCA average and R19 are generally non-significant, in that the confidence intervals overlap. This is again true for both the LOCA average as well as the individual models, where at most eight of the solutions have significantly different trends from R19 (with Tyndall Air Force Base being the lone exceptions; see the strip text of Figure 201.).

Taking a step back, we next explore how these considerations play out across the other facilities shown in Figure 1 and in all seasons. First, across all locations and seasons, the percent differences in return values between the LOCA average and R19 are approximately constant across event rarity: over $70 \%$ of locations have non-significant trends in the percent difference as a function of return period, and the other $30 \%$ (which are techically significant) only change by $\approx-2 \%$ per 10 -year increase in return period. As a result, we again focus on the 20 -year return value. Figure 3 a) shows the number of LOCA solutions with significantly different 20-year return values, where it is clear that across locations and seasons almost all of the solutions (approximately 25-27) are significantly different than R19. The magnitude 
of this difference is summarized in Figure 3 (b), which shows the percent difference between R19 and the LOCA average with black circles to identify locations for which the difference is significant. As with the case study locations, these differences are significant nearly everywhere, ranging between $-25 \%$ and $-50 \%$. Finally, Figure 3 (c) shows the number of LOCA solutions for which the 65-year (1951-2005) linear trend differs from R19. Again, as we saw in the case study, very few solutions have trends that differ significantly from R19, on average four across each season.

In summary, using these facilities as a case study, in all seasons the LOCA solutions have significant low biases in daily extreme precipitation return value estimates relative to R19, although the low biases are roughly consistent across return period. Estimates of trends in extremes from both R19 and LOCA have very large uncertainties and do not appear to significantly differ from one another.

\subsection{Reasons for large discrepancies in LOCA return values}

In Section 3.1. we found that daily precipitation return value estimates from the LOCAdownscaled models at the locations of interest are approximately $25 \%$ to $50 \%$ too small relative to R19. As shown in Pierce et al. (2021), similar biases exist for the L15 training data relative to R19. Moreover, the LOCA solutions match the training data with respect to return value estimates and trends in extremes (see Section 1 of the Supplemental Materials). This latter finding is not surprising, since the L15 product was used to train the LOCA downscaling procedure and that procedure is dominated by values from the observations set. However, the differences between, specifically, L15 and R19 are concerning since the low biases in estimates of extreme precipitation from L15 relative to R19 have been propagated through to the downscaled solutions for return periods if not for space- and time-averaged distributions of extremes. The natural follow-up question is: why? We now show that there are two primary reasons for these differences: (1) a time-of-day correction in L13/L15, and (2) the application of gridding to fields of daily precipitation. We now examine each of these reasons in turn. In light of the fact that horizontal resolution is not the dominant driver of the L15 low bias in daily precipitaton extremes (see Section 2 of the Supplemental Materials), in order to compare L15 and R19 in we regrid the L15 return values to the $1 / 4^{\circ} \mathrm{R} 19$ grid via a nearest-neighbor scheme that averages the four L15 nearest-neighbors to each R19 centroid.

Time-of-day correction in L15 As described in Pierce et al. (2021), the weather station data used to create L15 are adjusted in order to "standardize" the daily data to all have the same measurement time. This adjustment is conducted prior to gridding, and is done as follows: define $P^{h}(t)$ to be a measurement of precipitation representing one day $t$, taken at hour $h=$ $0, \ldots, 23$. In L15, these values are time-adjusted such that all stations have a measurement time of midnight $(h=23)$ as follows:

$$
P^{23}(t)=P^{h}(t) \Delta+P^{h}(t+1)(1-\Delta),
$$

where $\Delta=(h+1) / 24$ (for further details, see Pierce et al. 2021). Pierce et al. (2021) show that the most common gauge observation times are between 6-8 AM local time; applying the time-adjustment means that for such measurements, the precipitation for day $t$ is mostly added to the measurement on day $t+1$. More importantly, Pierce et al. (2021) show that the time adjustment systematically reduces seasonal maxima (except in very rare situations), 
while also increasing the fraction of wet days and decreasing the average precipitation on wet days (these latter two issues are less relevant for the study at hand but are nonetheless important). Pierce et al. (2021) show that, averaged over CONUS, L15 underestimates 20year return values by approximately $26 \%$ in DJF and $32 \%$ in JJA (similar to what we find in this paper). In light of this known feature of the L15 data processing algorithm, Pierce et al. (2021) develop a new version of the L15 data that does not include the time adjustment, henceforth "L15-unadj". Applying the same GEV analysis to L15-unadj significantly reduces the low biases in 20-year return values, to just $4 \%$ in DJF and $12 \%$ in JJA.

However, taking a closer look at Figure 5 in Pierce et al. (2021), it appears as though both of these CONUS-average low biases (for L15 and L15-unadj) are likely larger than they should be due to the inclusion of areas of complex topography in the western United States. In these areas, the L15 and L15-unadj return values are actually significantly larger than R19 (by as much as $+30 \%$ ). This is almost certainly due to the different ways each data set accounts for elevation: in R19, a simple linear elevation scaling is applied to the GEV parameter fields, while (as previously mentioned) L15 and L15-unadj use a PRISMbased adjustment (Daly et al. 1994) that incorporates other topographic variables. In light of these important differences and the difficulties of modeling precipitation in areas of complex topography (which are beyond the scope of this paper), we limit our comparison to CONUS grid cells with an elevation of less than 1500 meters above sea level. We note that these problems may be influenced more by elevation gradients as opposed to absolute elevation, but using an absolute threshold is a simple way to navigate the challenges of modeling precipitation in places with complex topography.

Gridding of daily data As was previously mentioned, a number of recent papers have documented how gridded daily products of precipitation (such as L15) are an inappropriate data source for characterizing extremes locally since daily precipitation exhibits fractal scaling and any spatial averaging will reduce extremes (Lovejoy et al. 2008, Maskey et al. 2016). To explicitly evaluate the magnitude of this reduction in extremes, we take the same input data as R19 (daily measurements of precipitation from 5,202 GHCN stations over 19502017) and apply bilinear interpolation (like the SYMAP algorithm used by L15) to convert the spatially irregular measurements to a regular $0.25^{\circ}$ grid-the same grid as the one used in R19. Then, at each grid cell, we extract the seasonal maximum daily precipitation in each year and fit the GEV distribution defined in (1) with a linear trend in the location parameter; we then calculate the maximum likelihood estimate of the 20 -year return value at each grid cell. While this is an admittedly simple interpolation scheme that does not make adjustments for complex topography, it allows us to isolate the specific influence of applying a standard gridding scheme to a fractal field. And, as mentioned in the previous paragraph, the facility locations under consideration in this paper are not in areas of complex topography.

Note that this procedure can be seen as reversing the order of operations in R19: in R19, one first calculates extreme statistics from the station data and then applies gridding, while here (as with L15) we instead first apply gridding to the daily station data and then calculate extreme statistics from the gridded result. As such, we denote this approach as "GHCNgrid-then-fit" since it uses GHCN station data as input but grids the daily data before fitting an extreme value distribution. And, importantly, this yields an apples-to-apples comparison since it uses the same input data as R19 and simply reverses the order of operations.

Decomposition of low extreme daily precipitation bias in $L 15$ Considering all $0.25^{\circ}$ grid cells in CONUS with an elevation of less than $1500 \mathrm{~m}$ (to avoid the influence of the PRISM adjustment in L15 and L15-unadj), we calculate the percent biases for L15, L15-unadj, and 
GHCN-grid-then-fit versus the R19 ground truth return values. Using these biases, we can explicitly quantify the contribution of the time-of-day (TOD) adjustment and the gridding of daily data to the total bias in L15. First, the contribution of the TOD adjustment can be calculated by taking the percent bias from L15 minus the percent bias in L15-unadj divided by the $\mathrm{L} 15$ bias, i.e.,

$$
\text { Fraction of bias due to TOD adjustment }=\frac{\operatorname{Bias}(\mathrm{L} 15)-\operatorname{Bias}(\mathrm{L} 15-\text { unadj })}{\operatorname{Bias}(\mathrm{L} 15)},
$$

where $\operatorname{Bias}(\cdot)$ is the average percent difference in 20 -year return values of the indicated data set relative to R19. Next, the contribution of gridding daily data can be calculated by directly comparing the two biases

$$
\text { Fraction of bias due to gridding daily data }=\frac{\operatorname{Bias}(\mathrm{GHCN} \text {-grid-then-fit })}{\operatorname{Bias}(\mathrm{L} 15)},
$$

since the primary difference between the GHCN-grid-then-fit and R19 data sets is the order of operations. The total percent bias in 20-year return values for L15 relative to R19 averaged over all $0.25^{\circ}$ CONUS grid cells with an elevation less than $1500 \mathrm{~m}$ is shown in Table 1 . Now that we have removed grid cells with complex topography (albeit rather crudely), we can see that the overall bias in L15 is slightly worse than was found in Pierce et al. (2021): the L15 return values are approximately $30 \%$ too small for DJF, MAM, and SON, and $>35 \%$ too small in JJA. The largest fraction of this bias is due to the time-of-day adjustment in L15, which accounts for $53 \%$ of the bias in JJA and up to $74 \%$ of the bias in DJF. The gridding of daily data has a lesser but nonetheless significant influence on the return values, accounting for about $35 \%$ of the bias in DJF to $50 \%$ of the bias in JJA. Clearly, the two rightmost columns of Table 1 do not add up to 1 , which indicates that there are some overlapping contributions to the bias from the TOD adjustment and gridding of daily data. Nonetheless, the fact that the sum of these two fractional contributions is close to 1 (actually 1.04 to 1.11) provides confidence that we are partitioning the bias into these two causes approximately correctly, especially since the return values from the different data sources (L15, L15-unadj, and GHCN-grid-then-fit) were calculated independently of one another.

\subsection{Scaling factors for adjusting LOCA biases}

When a user is interested in using the LOCA solutions for estimating return values, the results presented in this paper can be used to "bias correct" LOCA return values by way of a simple scaling factor. Given that 1951-2005 linear trends in return values for LOCA are generally indistinguishable from corresponding trends in R19 and differences are consistent across return period (see Section 3.1), a time- and return period-invariant scaling factor can be derived by calculating the ratio between $\mathrm{L} 15$ and R19 return values as

$$
\mathrm{sf}_{\mathbf{g}}=\frac{\widehat{\phi}_{2005,20}^{\mathrm{R} 19}(\mathbf{g})}{\widehat{\phi}_{2005,20}^{\mathrm{L} 15}(\mathbf{g})}
$$

(of course, the scaling factors depend on season as well), where $\mathbf{g}$ corresponds to the $1 / 16^{\circ}$ grid used in L15 and LOCA and we arbitrarily choose the year 2005 and the 20-year return period. The idea here is that if one estimates grid-cell $r$-year return values for the LOCA solutions in exactly the same way as described in Section 2.2. one could multiply the return 
value by sf $\mathrm{g}$ to yield return values that have the same climatology as R19. Furthermore, the scaling factors are based on L15 since biases present in the predictant of a statistical downscaling solution will also be present in the output (Maraun et al. 2010). Such a correction provides a path forward for users who require the daily data and range of solutions provided by the LOCA-downscaled CMIP5 GCMs but still wish to estimate pointwise return values. The seasonal scaling factors based on the percent differences are provided as a publicly available data product; see Risser et al. (2021).

As a simple example of how this correction works, we apply the scaling factor to the wintertime LOCA return values at two sample sites, Tyndall Air Force Base and Fort Hood (see Figure 1). Recall from Figure 2 that the return values from LOCA are too small relative to R19 for both sites, but much more so at Tyndall Air Force Base. The scaling factors for Tyndall and Fort Hood are 1.67 and 1.33, reflecting the larger (low) bias at Tyndall. The adjusted return values for these two sites are shown in Figure 4 , where we also show estimates from R19 and L15 for reference. The plotted lines for LOCA and LOCA-adjusted represent the multi-model mean and the confidence band shows the multi-model spread. At both sites, the adjusted return value curves agree much more closely with the R19 estimates, with the uncertainty bands overlapping in almost all cases. Our main point is that the adjusted LOCA return values could be used by practitioners. Given the well-documented difficulties in bias correcting downscaling projections of daily precipitation (Turco et al. 2017), these scaling factors could furthermore be applied to projected downscaling solutions, allowing practitioners to assess both relative and absolute changes in extreme precipitation return values under future emissions scenarios. However, an assessment of future projections is beyond the scope of this current work.

\section{Discussion}

Our findings in this paper show how construction of the training data set can influence key aspects of statistically downscaled solutions, and illustrate the utility of evaluating extreme statistics by using a product specifically designed for that purpose via extreme value analysis. These results echo downscaling intercomparison efforts that pointed to the central importance of training data (Gutmann et al. 2012, 2014). Other training data sets that do not incorporate the time-of-day correction may have smaller biases, but biases due to the gridding of daily data will persist in the absence of more sophisticated gridding techniques that do not over-smooth extreme values and variability.

To demonstrate the utility of our findings regarding representations of observed precipitation extremes, we provide a data product consisting of scaling factors that effectively correct the lower biases that we identify in LOCA return value estimates relative to a dedicated precipitation extremes data set. In this way we intend to support practitioners and other endusers of LOCA solutions if and as they use these datasets for location-specific analysis of extreme daily precipitation (Risser et al. 2021, in preparation). These correction factors can also be applied to other downscaled products that are trained on L15, after accounting for the corresponding grid differences, and the findings can potentially serve as a guide to develop correction factors for other statistically-downscaled products where training datasets are shown to exhibit biases in extreme behavior. We recognize that many downscaling approaches employ a range of bias-correction techniques out of necessity (Christensen et al. 2008, Mearns et al. 2012, Sillmann et al. 2013), though not without controversy (Ehret et al. 2012). The results presented indicate that an additional bias-correction for extreme precipitation is needed for historically-downscaled products. We did not explore whether 
such a bias-correction would be needed for extremal values in future projections, but at least where statistical methods are used for downscaling future projections, such extremal values will need to be adjusted, and the approach and dataset presented here can provide a starting point to inform such an analysis. We note that even though the difference in trends between LOCA and R19 are not statistically significant (at the $95 \%$ confidence level), the difference in percent changes in the future may still be different.

While the focus of this particular analysis is on L15 and LOCA, any statistical downscaling method trained with L15 or using a similar time-of-observation correction scheme without correcting for this bias would be expected to show similar results. How observational training data sets are constructed can affect particular aspects of a downscaling solution, and using the alternative method of direct extreme value analysis of the variables of interest can add valuable information for local resilience planning. We specifically note that these low biases in daily extremes for L15 do not result in low (or dry) biases at longer timescales (e.g., multi-day or monthly). As described below, extreme daily precipitation values are diluted by being distributed across space (via gridding) and time (by a time-of-observation adjustment). Multi-day, seasonal, and annual precipitation totals are preserved by this "smearing" even as daily extremes are reduced.

The low bias in precipitation return values in commonly used gridded products of the historical record may be problematic for decisions regarding facility resource management. One common approach to using downscaled solutions, which recognizes the challenges with using the absolute values of those solutions, is to look at the relative change between the simulated historical record and a future projection in a given quantile (e.g. Cannon et al., 2015). We show that making decisions based on relative change in extreme precipitation at a given location will be problematic, since the relative change in extreme precipitation will propagate the low-bias in extreme precipitation that we have shown can exist in downscaling solutions from the historical record.

Whenever multiple techniques can be considered for a common problem (here, an observational data set for assessing daily precipitation) a trade-off is typically involved. For example, the special-purpose precipitation extremes product R19 does not provide daily time series of precipitation and so cannot be used as a substitute for daily precipitation fields in all applications including ones to train statistical downscaling methods. Conversely, the standard daily gridded product and others like it cannot capture extremes as well as the dedicated product based on extreme value analysis. Depending on the application, using one or the other product (daily gridded or seasonal extreme measures) may be preferable, but we show that using aspects of both can produce more useful results than using either singly. We note that although we do not pursue the question in this study, we consider that gridded daily precipitation data products are appropriate for conducting GCM model evaluation (see, e.g., Chen and Knutson 2008, Gervais et al., 2014, Risser and Wehner, 2020, Wehner et al. 2021); on the other hand, products such as Risser et al. (2019c a) are the appropriate for considering pointwise applications, and are therefore used as the standard of comparison in this paper.

More broadly, this paper explores a thorny issue for downscaling: products that are developed for grid boxes at $6 \mathrm{~km}$, which are very small relative to the parent model, may not be the most informative for analyses of extreme precipitation at other spatial scales. To be clear, this problem is based on the technique used to generate the training data set, and not due to the size of the downscaled grid box. Fundamentally, daily precipitation is a fractal field, and the gridding of that field produces artifacts. While the biases we identified here may decrease with the decreasing size of the grid boxes in the application domain, users of gridded observational data should exercise caution when using gridded datasets for analyz- 
ing point extremes of these environmental variables. Our results show the additional value that can be gained by considering results from a targeted extreme value analysis in addition to using statistics calculated from a daily gridded product. With respect to extreme precipitation, a correction is required to conventionally gridded products in order to incorporate unbiased estimates of extreme precipitation into location-specific analyses. While a correction factor may address gridding biases, the findings here show how attention must be paid to the corruption of extrema information from gridding observational data. The bias induced from gridding in the manner explored here can be especially troublesome where changes in extreme precipitation need to be estimated. Baseline analyses of models and observational products over the historical period form a foundation for analyzing extreme precipitation in future climate. Regardless of whether location-specific analyses use future projections with statistical, dynamical, or hybrid methods to incorporate nonstationarity into their downscaled solutions, those analyses need to begin with information from historical data that is unbiased from gridding methods.

\section{Declarations}

Funding Funding sources blinded.

Conflicts of interest/Competing interests The authors declare no competing interests.

Availability of data and material The LOCA solutions used in this paper are publicly available atftp://gdo-dcp.ucllnl.org/pub/dcp/archive/cmip5/loca/LOCA_ 2016-04-02/, the Livneh et al. (2015b) data are publicly available at ftp://ftp. nodc.noaa.gov/nodc/archive/arc0077/0129374/1.1/data/0-data/daily/ The Risser et al. (2019c) data product is publicly available at https://doi.org/10. $7910 /$ DVN/LULNUQ

Code availability Not applicable.

\section{References}

Abatzoglou JT, Brown TJ (2012) A comparison of statistical downscaling methods suited for wildfire applications. International Journal of Climatology 32(5):772-780

Ayar PV, Blanchet J, Paquet E, Penot D (2020) Space-time simulation of precipitation based on weather pattern sub-sampling and meta-gaussian model. Journal of Hydrology 581:124451

Barsugli JJ, Guentchev G, Horton RM, Wood A, Mearns LO, Liang XZ, Winkler JA, Dixon K, Hayhoe K, Rood RB, et al. (2013) The practitioner's dilemma: How to assess the credibility of downscaled climate projections. Eos, Transactions American Geophysical Union 94(46):424-425

Brown C, Ghile Y, Laverty M, Li K (2012) Decision scaling: Linking bottom-up vulnerability analysis with climate projections in the water sector. Water Resources Research 48(9)

Cannon AJ, Sobie SR, Murdock TQ (2015) Bias correction of gcm precipitation by quantile mapping: How well do methods preserve changes in quantiles and extremes? Journal of Climate 28(17):6938-6959 
Chen CT, Knutson T (2008) On the verification and comparison of extreme rainfall indices from climate models. Journal of Climate 21(7):1605-1621

Chen J, Zhang XJ (2021) Challenges and potential solutions in statistical downscaling of precipitation. Climatic Change 165(3):1-19

Christensen JH, Boberg F, Christensen OB, Lucas-Picher P (2008) On the need for bias correction of regional climate change projections of temperature and precipitation. Geophysical Research Letters 35(20)

Coles S (2001) An Introduction to Statistical Modeling of Extreme Values. Lecture Notes in Control and Information Sciences, Springer, URL https://books.google.com/ books? id=2 nugUEaKqFEC

Daly C, Neilson RP, Phillips DL (1994) A statistical-topographic model for mapping climatological precipitation over mountainous terrain. Journal of applied meteorology 33(2):140-158

Daly C, Halbleib M, Smith JI, Gibson WP, Doggett MK, Taylor GH, Curtis J, Pasteris PP (2008) Physiographically sensitive mapping of climatological temperature and precipitation across the conterminous united states. International Journal of Climatology: a Journal of the Royal Meteorological Society 28(15):2031-2064

Ehret U, Zehe E, Wulfmeyer V, Warrach-Sagi K, Liebert J (2012) Hess opinions" should we apply bias correction to global and regional climate model data?". Hydrology \& Earth System Sciences Discussions 9(4)

Engle NL (2011) Adaptive capacity and its assessment. Global environmental change 21(2):647-656

Eyring V, Bony S, Meehl GA, Senior CA, Stevens B, Stouffer RJ, Taylor KE (2016) Overview of the Coupled Model Intercomparison Project Phase 6 (CMIP6) experimental design and organization. Geoscientific Model Development 9(5):1937-1958

Gervais M, Tremblay LB, Gyakum JR, Atallah E (2014) Representing extremes in a daily gridded precipitation analysis over the United States: Impacts of station density, resolution, and gridding methods. Journal of Climate 27(14):5201-5218

Giorgi F, Gutowski Jr WJ (2015) Regional dynamical downscaling and the CORDEX initiative. Annual Review of Environment and Resources 40:467-490

Gutmann E, Pruitt T, Clark MP, Brekke L, Arnold JR, Raff DA, Rasmussen RM (2014) An intercomparison of statistical downscaling methods used for water resource assessments in the United States. Water Resources Research 50(9):7167-7186

Gutmann E, Barstad I, Clark M, Arnold J, Rasmussen R (2016) The intermediate complexity atmospheric research model (ICAR). Journal of Hydrometeorology 17(3):957-973

Gutmann ED, Rasmussen RM, Liu C, Ikeda K, Gochis DJ, Clark MP, Dudhia J, Thompson G (2012) A comparison of statistical and dynamical downscaling of winter precipitation over complex terrain. Journal of Climate 25(1):262-281

Hallegatte S (2009) Strategies to adapt to an uncertain climate change. Global environmental change 19(2):240-247

King AD, Alexander LV, Donat MG (2013) The efficacy of using gridded data to examine extreme rainfall characteristics: a case study for Australia. International Journal of Climatology 33(10):2376-2387

Klemes V, et al. (1982) Empirical and causal models in hydrology. Scientific basis of water resource management pp 95-104

Livneh B, Rosenberg EA, Lin C, Nijssen B, Mishra V, Andreadis KM, Maurer EP, Lettenmaier DP (2013) A long-term hydrologically based dataset of land surface fluxes and states for the conterminous United States: Update and extensions. Journal of Climate 26(23):9384-9392 
Livneh B, Bohn TJ, Pierce DW, Munoz-Arriola F, Nijssen B, Vose R, Cayan DR, Brekke L (2015a) A spatially comprehensive, hydrometeorological data set for Mexico, the US, and Southern Canada 1950-2013. Scientific data 2(1):1-12

Livneh B, Bohn TJ, Pierce DW, Munoz-Arriola F, Nijssen B, Vose R, Cayan DR, Brekke L (2015b) A spatially comprehensive, hydrometeorological data set for Mexico, the US, and Southern Canada (NCEI Accession 0129374). NOAA National Centers for Environmental Information Dataset (Daily precipitation) DOI https://doi.org/10.7289/v5x34vf6, accessed April 13, 2020.

Lopez-Cantu T, Prein AF, Samaras C (2020) Uncertainties in Future US Extreme Precipitation From Downscaled Climate Projections. Geophysical Research Letters 47(9):e2019GL086797

Lovejoy S, Schertzer D, Allaire V (2008) The remarkable wide range spatial scaling of TRMM precipitation. Atmospheric Research 90(1):10-32, DOI 10.1016/j. atmosres.2008.02.016, URL http: // linkinghub.elsevier.com/retrieve/ pii/S0169809508000562

Maraun D, Wetterhall F, Ireson A, Chandler R, Kendon E, Widmann M, Brienen S, Rust H, Sauter T, Themeß1 M, et al. (2010) Precipitation downscaling under climate change: Recent developments to bridge the gap between dynamical models and the end user. Reviews of geophysics $48(3)$

Martinich J, Crimmins A (2019) Climate damages and adaptation potential across diverse sectors of the united states. Nature climate change 9(5):397-404

Maskey ML, Puente CE, Sivakumar B, Cortis A (2016) Encoding daily rainfall records via adaptations of the fractal multifractal method. Stochastic Environmental Research and Risk Assessment 30(7):1917-1931, DOI 10.1007/s00477-015-1201-7, URL http: //link.springer.com/10.1007/s00477-015-1201-7

Mearns LO, Arritt R, Biner S, Bukovsky MS, McGinnis S, Sain S, Caya D, Correia Jr J, Flory D, Gutowski W, et al. (2012) The North American regional climate change assessment program: overview of phase I results. Bulletin of the American Meteorological Society 93(9):1337-1362

Menne MJ, Durre I, Vose RS, Gleason BE, Houston TG (2012) An overview of the Global Historical Climatology Network-Daily database. Journal of Atmospheric and Oceanic Technology 29(7):897-910

Milly P, Betancourt J, Falkenmark M, Hirsch RM, Kundzewicz ZW, Lettenmaier DP, Stouffer RJ (2008) Stationarity is dead: Whither water management? Earth 4:20

Moss RH (2017) Nonstationary Weather Patterns and Extreme Events Informing Design and Planning for Long-Lived Infrastructure. Tech. rep., Workshop Report on Nonstationarity, ESTCP Project RC-201591, URL https://www.serdp-estcp.org/

News-and-Events/Conferences-Workshops/Past-RC-Workshops/ Nonstationary-Weather-Patterns-and-Extreme-Events-2017

Newman AJ, Clark MP, Craig J, Nijssen B, Wood A, Gutmann E, Mizukami N, Brekke L, Arnold JR (2015) Gridded ensemble precipitation and temperature estimates for the contiguous United States. Journal of Hydrometeorology 16(6):2481-2500

Paciorek C (2016) climextRemes: Tools for Analyzing Climate Extremes. URL https: //CRAN.R-project. org/package $=$ climextRemes, $r$ package version 0.1.2

Paciorek C, Stone D, Wehner M (2018) Quantifying statistical uncertainty in the attribution of human influence on severe weather. Weather and Climate Extremes 20:69-80, DOI 10.1016/j.wace.2018.01.002

Pierce DW, Cayan DR, Thrasher BL (2014) Statistical downscaling using localized constructed analogs (LOCA). Journal of Hydrometeorology 15(6):2558-2585 
Pierce DW, Su L, Cayan DR, Risser MD, Livneh B, Lettenmaier DP (2021) An extremepreserving long-term gridded daily precipitation data set for the conterminous United States. Journal of Hydrometeorology

Risser M, Paciorek C, Wehner M, O’Brien T, Collins W (2019a) A probabilistic gridded product for daily precipitation extremes over the United States. Harvard Dataverse DOI 10.7910/DVN/LULNUQ, URL/https: / / doi .org/10.7910/DVN/LULNUQ

Risser M, Feldman D, Wehner M, Pierce D, Cayan D, Arnold J (2021) Scaling factors for improving resilience to extreme daily precipitation events for the L15 data product and LOCA solutions. Harvard Dataverse DOI pending, URL TBD

Risser MD, Wehner MF (2020) The effect of geographic sampling on evaluation of extreme precipitation in high-resolution climate models. Advances in Statistical Climatology, Meteorology and Oceanography 6(2):115-139

Risser MD, Paciorek CJ, O'Brien TA, Wehner MF, Collins WD (2019b) Detected changes in precipitation extremes at their native scales derived from in situ measurements. Journal of Climate 32(23):8087-8109, DOI 10.1175/JCLI-D-19-0077.1, URL https : //doi.org/10.1175/JCLI-D-19-0077.1,https://doi.org/10.1175/ JCLI-D-19-0077.1

Risser MD, Paciorek CJ, Wehner MF, O'Brien TA, Collins WD (2019c) A probabilistic gridded product for daily precipitation extremes over the United States. Climate Dynamics 53(5):2517-2538, DOI 10.1007/s00382-019-04636-0, URL https : / / doi .org/ $10.1007 / \mathrm{s} 00382-019-04636-0$

Shepard D (1968) A two-dimensional interpolation function for irregularly-spaced data. In: Proceedings of the 1968 23rd ACM national conference, pp 517-524

Shepard DS (1984) Computer mapping: The SYMAP interpolation algorithm. In: Spatial statistics and models, Springer, pp 133-145

Sillmann J, Kharin V, Zhang X, Zwiers F, Bronaugh D (2013) Climate extremes indices in the cmip5 multimodel ensemble: Part 1. model evaluation in the present climate. Journal of Geophysical Research: Atmospheres 118(4):1716-1733

Stoner AM, Hayhoe K, Yang X, Wuebbles DJ (2013) An asynchronous regional regression model for statistical downscaling of daily climate variables. International Journal of Climatology 33(11):2473-2494

Taylor KE, Stouffer RJ, Meehl GA (2012) An overview of CMIP5 and the experiment design. Bulletin of the American Meteorological Society 93(4):485-498

Timmermans B, Wehner M, Cooley D, O’Brien T, Krishnan H (2019) An evaluation of the consistency of extremes in gridded precipitation data sets. Climate Dynamics 52(11):6651-6670, DOI 10.1007/s00382-018-4537-0, URLhttps : / / doi .org/10 . $1007 / \mathrm{s} 00382-018-4537-0$

Turco M, Llasat MC, Herrera S, Gutiérrez JM (2017) Bias correction and downscaling of future $\mathrm{rcm}$ precipitation projections using a mos-analog technique. Journal of Geophysical Research: Atmospheres 122(5):2631-2648

Vano JA, Arnold JR, Nijssen B, Clark MP, Wood AW, Gutmann ED, Addor N, Hamman J, Lehner F (2018) Dos and don'ts for using climate change information for water resource planning and management: guidelines for study design. Climate Services 12:1-13

Vrac M, Stein M, Hayhoe K (2007) Statistical downscaling of precipitation through nonhomogeneous stochastic weather typing. Climate Research 34(3):169-184

Wehner MF, Lee J, Risser MD, Ullrich P, Gleckler P, Collins WD (2021) Evaluation of extreme subdaily precipitation in high-resolution global climate model simulations. The

Philosophical Transactions of the Royal Society 379, URL https://doi . org/10 . $1098 /$ rsta.2019.0545 
Wood AW, Leung LR, Sridhar V, Lettenmaier D (2004) Hydrologic implications of dynamical and statistical approaches to downscaling climate model outputs. Climatic change 62(1):189-216

Wuebbles DJ, Fahey DW, Hibbard KA (2017) Climate science special report: fourth national climate assessment, volume I 
Table 1 Total percent bias in 20-year return values for L15 relative to R19 (second column from the left) averaged over all $0.25^{\circ}$ CONUS grid cells with an elevation less than $1500 \mathrm{~m}$. The fraction of the bias due to both the time-of-day (TOD) adjustment and the gridding of daily data are also shown in the two rightmost columns.

\begin{tabular}{lccc}
\hline Season & Total bias (L15) & Frac. of bias due to TOD adj. & Frac. of bias due to gridding daily data \\
\hline DJF & $-29.4 \%$ & 0.74 & 0.37 \\
MAM & $-30.9 \%$ & 0.63 & 0.43 \\
JJA & $-35.7 \%$ & 0.53 & 0.51 \\
SON & $-30.4 \%$ & 0.63 & 0.41 \\
\hline
\end{tabular}

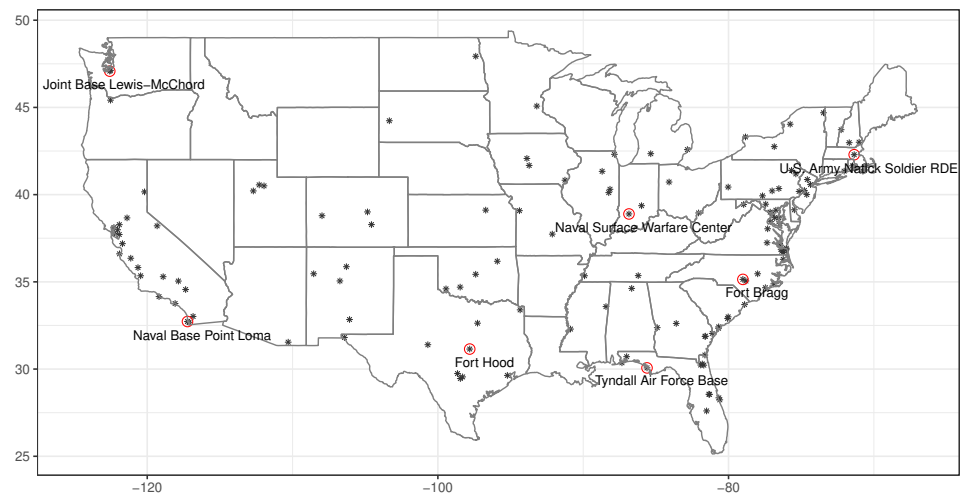

Fig. 1 Geographic distribution of the $163 \mathrm{DoD}$ facilities considered in this study (geocoded based on their ZIP code) with the seven facilities used for the case study in Section 3 
(a) DJF return values for $2005(\mathrm{~mm})$

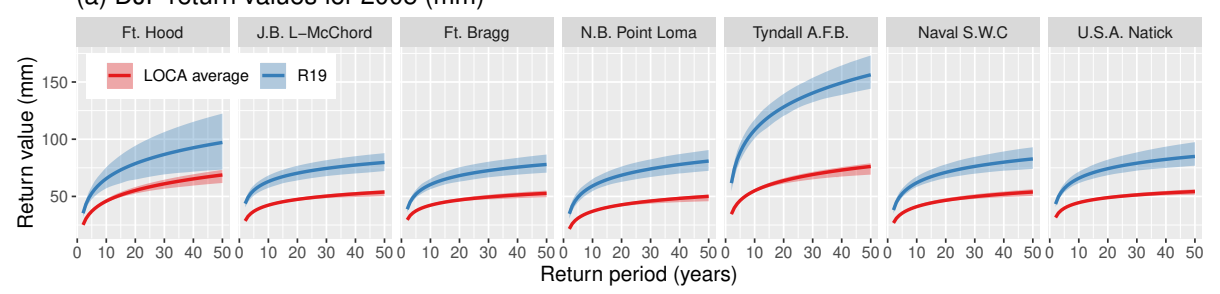

(b) Difference between LOCA average and R19 (\%)

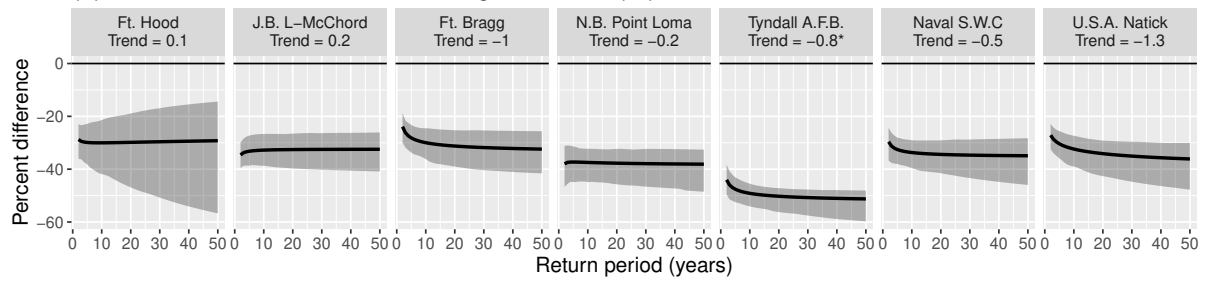

(c) Number of LOCA solutions (of 31) significantly different from R19

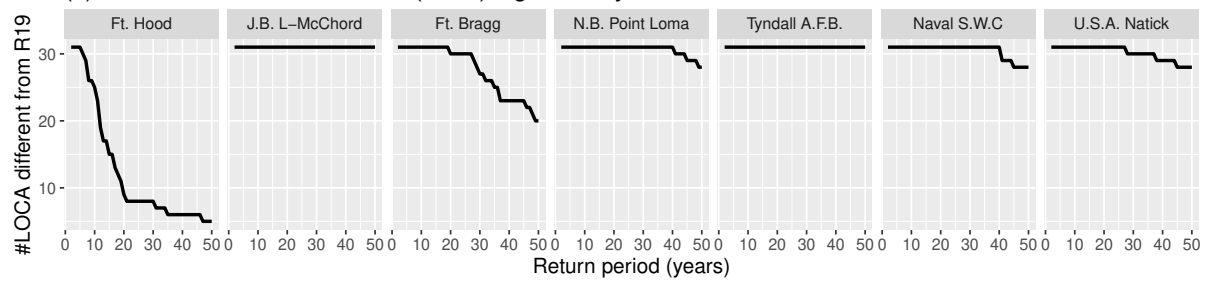

(d) 65-year (1951-2005) linear trends in 20-year return values

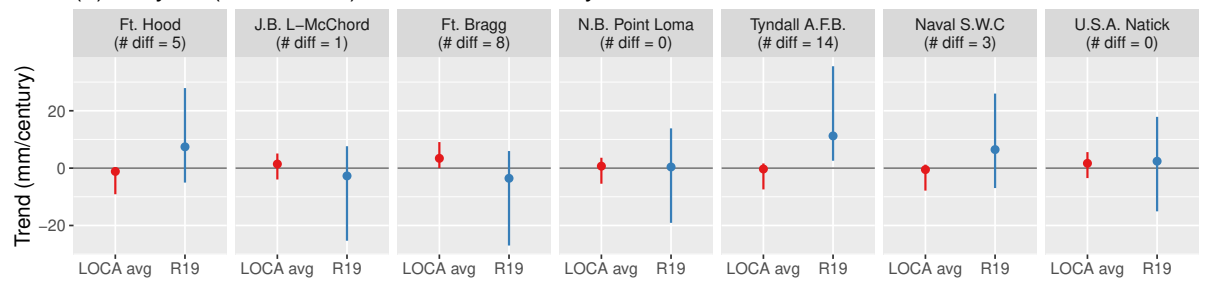

Fig. 2 Best estimates and 95\% basic bootstrap confidence intervals of the $r$-year return values of Rx1Day (panel a) and percent differences (panel b) for each of seven example facilities, comparing the Risser et al. (2019c) product (R19) and the average of the 31 LOCA-downscaled CMIP5 models. Panel c. shows the number of individual LOCA solutions (31 total) that differ significantly from R19 for each return period. 
(a) Number of LOCA solutions (of 31) with significantly different 20 -year return values

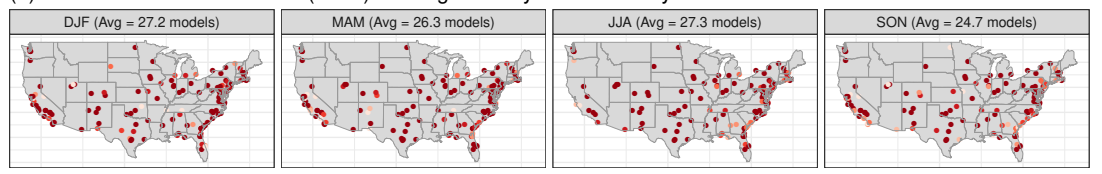

(b) Percent difference in 20-year return values, LOCA average vs. R19

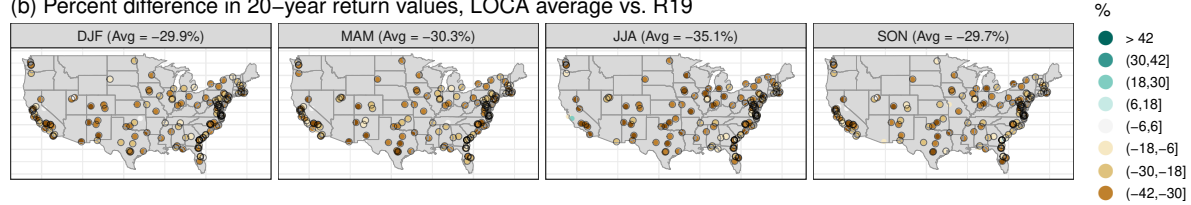

(c) Number of LOCA solutions (of 31) with significantly different return value trends

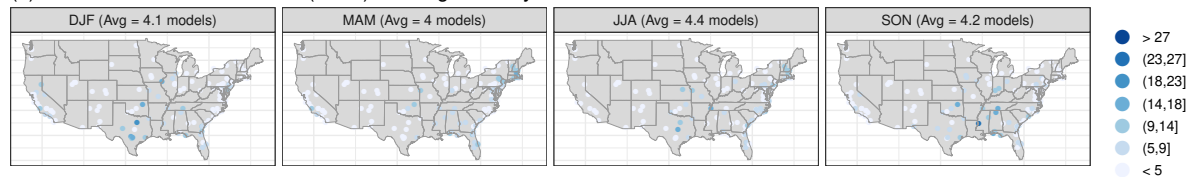

Fig. 3 The number of LOCA solutions with significantly different 20-year return value estimates versus R19 (panel a.; the average number of models is shown in the strip text), as well as the percent difference between the LOCA-average 20-year return value and the corresponding quantity from R19 (panel b.). Locations with a significant difference between R19 and the LOCA average are plotted with a black circle. Panel (c) shows the number of LOCA solutions for which the 65-year (1951-2005) linear trend in 20-year return value differs from the R19 trend (with the seasonal average number of solutions shown in the strip text).

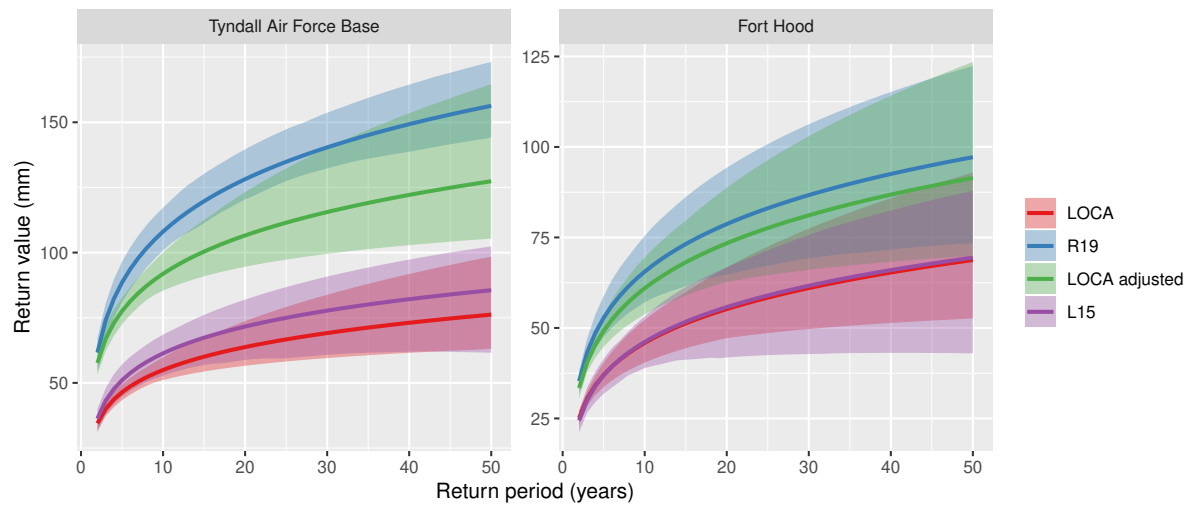

Fig. 4 Return values for R19 and the LOCA solutions, before after applying the location-specific scaling factor to LOCA at the Tyndall Air Force Base (scaling factor $=1.67$ ) and Fort Hood (scaling factor $=1.33$ ) grid cells. The L15 return values are also shown for reference (recall that the scaling factors are calculated as R19/L15). For R19 and L15, the 95\% basic bootstrap confidence band is shown; for the LOCA and LOCAadjusted, the band represents the multi-model spread (also, the plotted line is the multi-model mean). 


\section{Supplementary Files}

This is a list of supplementary files associated with this preprint. Click to download.

- supplementblind.pdf 